\title{
A systematic review of the efficacy comparison of acupuncture and traditional Chinese medicine in the treatment of primary dysmenorrhea
}

\author{
Jing Lin ${ }^{1,2}$, Wenyan Liao $^{3}$, Qiaoming $\mathrm{Mo}^{3}$, Peng Yang ${ }^{4}$, Xiaohong Chen ${ }^{4}$, Xiongjiang Wang ${ }^{4}$, Xian Huang ${ }^{2}$, \\ Dongming $\mathrm{Lu}^{2}$, Mingchen $\mathrm{Ma}^{4}$, Fengshi $\mathrm{He}^{4}$, Pingting $\mathrm{Wu}^{4}$, Kunyin $\mathrm{Li}^{5}$, Wantao Liang ${ }^{2}$, Hongliang Tang ${ }^{2}$ \\ ${ }^{1}$ Guangzhou University of Chinese Medicine, Guangzhou, China; ${ }^{2}$ The First Affiliated Hospital of Guangxi University of Chinese Medicine, \\ Nanning, China; ${ }^{3}$ Affiliated International Zhuang Medicine Hospital, Guangxi University of Chinese Medicine, Nanning, China; ${ }^{4}$ Guangxi \\ University of Chinese Medicine, Nanning, China; ${ }^{5}$ The Third Affiliated Hospital of Guangzhou University of Chinese Medicine, Guangzhou, China \\ Contributions: (I) Conception and design: J Lin, W Liang, H Tang; (II) Administrative support: W Liao, Q Mo, P Yang; (III) Provision of study \\ materials or patients: X Chen, X Wang, X Huang; (IV) Collection and assembly of data: D Lu, M Ma, F He, P Wu; (V) Data analysis and \\ interpretation: J Lin, W Liang, H Tang, K Li; (VI) Manuscript writing: All authors; (VII) Final approval of manuscript: All authors. \\ Correspondence to: Wantao Liang; Hongliang Tang. The First Affiliated Hospital of Guangxi University of Chinese Medicine, Nanning, China. \\ Email: liangwantao6688@163.com; tanghongliang@gxtcmu.edu.cn.
}

\begin{abstract}
Background: Dysmenorrhea is one of the most common symptoms in gynecology. It refers to people who experience lower abdominal pain, swelling, backache, or another discomfort before and after menstruation or during menstruation, which seriously affects the quality of life and work. Clinically, there are many methods to treat primary dysmenorrhea, among which acupuncture and traditional Chinese medicine (TCM) are beneficial. This work aimed to test the efficacy of acupuncture and TCM in the treatment of primary dysmenorrhea and hope to supply more reliable evidence for clinical treatment.

Methods: We searched articles from the Wanfang database and China National Knowledge Infrastructure and collected the clinical, randomized, or quasi-randomized controlled trials of acupuncture compared with TCM for primary dysmenorrhea. We tested the quality and data of the included studies according to the Cochrane criteria and compiled detailed systematic reviews and meta-analysis.

Results: Seven articles (including 492 patients) on acupuncture and TCM for primary dysmenorrhea were included in the systematic review. Seven studies were included in the final analysis, and there was no heterogeneity among the studies $\left(\mathrm{P}=0.98, \mathrm{I}^{2}=0 \%\right)$. The results showed that the clinical effectiveness of acupuncture was better than TCM (OR: 4.86, 95\% CI: 2.84-8.33, Z =5.75, P<0.00001).
\end{abstract}

Conclusions: The efficacy of acupuncture is superior to TCM in the treatment of primary dysmenorrhea.

Keywords: Primary dysmenorrhea; acupuncture; traditional Chinese medicine (TCM); systematic review

Submitted Jun 21, 2020. Accepted for publication Sep 25, 2020.

doi: 10.21037/apm-20-1734

View this article at: http://dx.doi.org/10.21037/apm-20-1734

\section{Introduction}

Dysmenorrhea is one of the most common problems in gynecology. It refers to an experience with lower abdominal pain, swelling, backache, or another discomfort before and after menstruation or during menstruation, which seriously affects the quality of life and work (1). Most of these patients are adolescent girls and unmarried young women (2). Clinically, it is divided into primary dysmenorrhea and secondary dysmenorrhea. The former refers to dysmenorrhea without pathological diseases in the reproductive system and accounts for over $90 \%$ of dysmenorrhea patients (3). The incidence of dysmenorrhea among women in China is $33.1 \%$, of which $53.2 \%$ are primary, and $13.5 \%$ of them are severely affected by 
dysmenorrhea in daily work or life (4). Epidemiological studies have shown that primary dysmenorrhea is a common gynecological disease and a common cause that affects women's routine work and quality of life. In some cases, primary dysmenorrhea pain leads to syncope or abuse of painkillers. Therefore, it is necessary to pay attention to the treatment of primary dysmenorrhea. There are many clinical methods for treating primary dysmenorrhea, among which acupuncture and traditional Chinese medicine (TCM) have the most significant effect. The Cochrane systematic review is to collect, test, and synthesize the ever-growing original clinical research data to maximize the inclusion of published and unpublished related research to avoid publication bias and to retrieve the overall conclusion of relevant interventions for clinical practice and health decision-making. By this method, we can supply valid and reliable evidence. Therefore, this study uses the Cochrane systematic evaluation method to compare the efficacy of acupuncture and TCM in the treatment of primary dysmenorrhea, hoping to supply more reliable evidence for clinical treatment. We present the following article in accordance with the PRISMA reporting checklist (available at http://dx.doi.org/10.21037/apm-20-1734).

\section{Methods}

\section{Criteria for eligibility}

Inclusion criteria: (I) publicly published randomized controlled trials or semi-randomized controlled trials, the primary language is Chinese; (II) research subjects are primarily dysmenorrhea patients, regardless of age; (III) the intervention measures of the experimental group are acupuncture, and the intervention measures of the control group are TCM; (IV) efficacy evaluation is divided into the cured, markedly effective, effective, and ineffective according to the "TCM Criteria for Diagnostic Efficacy of Diseases"; (V) high-quality research with low bias. Otherwise, if there is no high-quality research, low-quality research should be carefully included. Exclusion criteria: (I) repetitive study; (II) non-randomized controlled trial; (III) the acupuncture and Chinese medicine groups are not included in the study simultaneously; (IV) the efficacy standard is not clear, or there is no efficacy standard.

\section{Search strategy}

Subject heading approach: subject heading "dysmenorrhea," subtopic headings "acupuncture" and "traditional Chinese medicine," limited to humans. Free word approach: use "acupuncture," "Chinese herbal medicine," "Chinese patent medicine," and their derivative participles to search together with the subject words, respectively.

\section{Data extraction}

We searched for articles published from Jan 1994 to May 2020. The retrieval systems are CNKI, Wanfang Data, and Weipu Data. We collected the primary information and data of the included literature in a table, including the name of the literature, the author, the source of the literature, the number of cases with specific treatment measures, the methodological characteristics of the trial design, the random allocation plan is fragrant hiding, trial measures and comparison methods.

\section{Quality evaluation}

Evaluation criteria: the Jadad scale is used to test the methodological quality of the literature: (I) the generation of random sequence: random numbers generated by computer or similar methods ( 2 points), random experiments but no random allocation method is described (1 point), use alternate allocation methods including odd and even numbers $(0$ points); (II) randomized concealment: the medical center or department pharmacy controls the allocation plan, or uses containers with consistent serial numbers, on-site computer control, sealed opaque envelopes, or other so that clinicians and patients cannot predict the allocation sequence (2 points); only shows using a random number table or other random allocation plan (1 point); alternate allocation, case number, weekday number, open random number list, serial code envelope, and any measure that cannot prevent the predictability of the grouping ( 0 points); and not used ( 0 points); (III) blinding method: consistent use of placebo tablets or similar methods are used ( 2 points), the trial is stated as blinding method, but no description about method (1 point); No double-blind method or blind method is not appropriate, including a comparison of tablets and injections (0 points); (IV) withdrawal: describe the number and reasons for withdrawal (1 point), no withdrawal is described (0 points). Points of 1-3 were low-quality articles, 4-7 points were high-quality articles.

\section{Statistical method}

Meta-analysis of the included literature was performed 
Table 1 Basic information and quality of included studies

\begin{tabular}{|c|c|c|c|c|c|c|}
\hline Trials & \multicolumn{2}{|c|}{ Results (rate of effectiveness, \%) } & \multicolumn{4}{|c|}{ Literature evaluation } \\
\hline Ding et al. 1998 (5) & 95.83 & 83.33 & Random & Not mentioned & Not mentioned & 1 \\
\hline Fang et al. 2016 (6) & 96.15 & 89.66 & Random & Not mentioned & Not mentioned & 1 \\
\hline Zhang et al. 2013 (7) & 100 & 90 & Random & Not mentioned & Not mentioned & 2 \\
\hline Liu et al. 2013 (9) & 95 & 61.5 & Random & Not mentioned & Not mentioned & 1 \\
\hline Wang et al. 2002 (10) & 91.2 & 70.3 & Random & Not mentioned & Not mentioned & 1 \\
\hline Leng et al. 2012 (11) & 94 & 80 & Random & Not mentioned & Not mentioned & 2 \\
\hline
\end{tabular}

TCM, traditional Chinese medicine. Diagnostic criteria in each report are all TCM criteria for diagnostic efficacy of diseases.

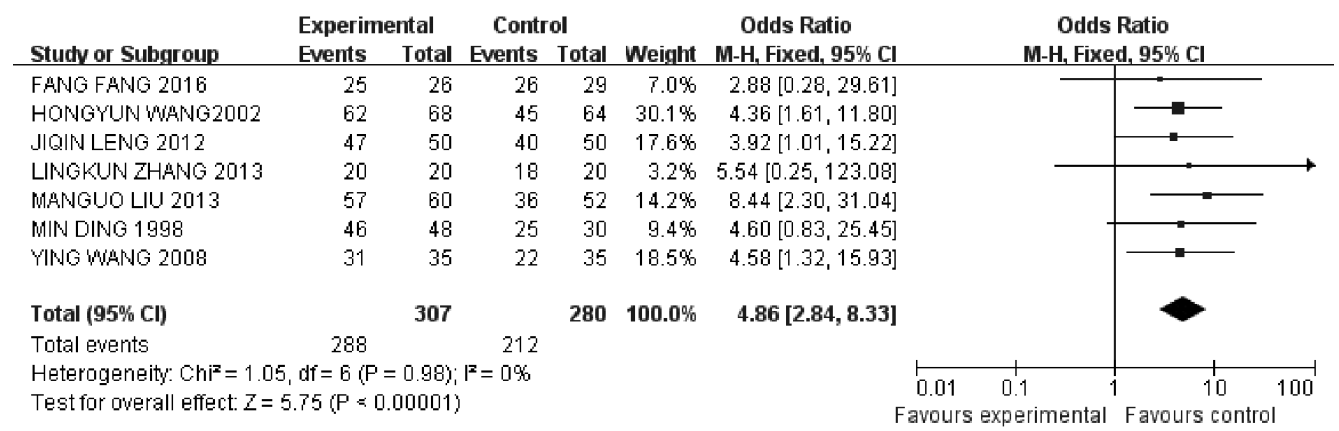

Figure 1 Comparison of the effects of acupuncture and traditional Chinese medicine.

using Cochrane Review Manager 5.1 software. The effect size of the binary variable data was expressed by relative risk (OR) and 95\% confidence interval (95\% CI). First, we tested the methodological heterogeneity and clinical heterogeneity of the included studies, and then test the statistical heterogeneity. Then, if the heterogeneity test result is $\mathrm{P}>0.1$ and $\mathrm{I}^{2}<50 \%$, then multiple similar studies can be homogeneous, and the fixed-effects model should be used for the meta-analysis. Further, when the heterogeneity test result has a result of $\mathrm{P} \leq 0.1$, multiple similar studies can be heterogeneous, and random effects models should be used.

\section{Results}

\section{The primary information of literature}

Two hundred-nine articles were retrieved (including 176 articles from CNKI, 12 articles from Wanfang, and 21 articles from Weipu). After preliminary screening, animal experiments and reviews were excluded. After reading the full text and checking with the RCT literature inclusion criteria, 12 randomized controlled trials were included in the quality evaluation. Then according to the requirements of evidence-based medicine literature, the control group of non-TCM studies, and literature reports, including unclear data and unclear diagnostic criteria, were excluded. Finally, seven pieces of research literature (5-11) were included. The basic situation is shown in Table 1 .

\section{A meta-analysis of the acupuncture group and TCM group}

Seven studies were included in the final analysis, and there was no heterogeneity among the studies $\left(\mathrm{P}=0.98, \mathrm{I}^{2}=0 \%\right)$, so the fixed effects model was used for meta-analysis. Combined effect size estimation according to the actual situation of the literature data, the meta-analysis of this study adopts the dichotomy type of count data for statistics, including the original data, effect size and combined effect size analysis results of each study, as shown in Figure 1 (effect size OR: 4.86, 95\% CI: 2.84-8.33, combined effect 
test $\mathrm{Z}=5.75, \mathrm{P}<0.00001$ ), the differences are statistically significant. The results show that the total clinical effective rate of acupuncture treatment of primary dysmenorrhea is better than the TCM control group.

\section{Discussion}

This study compared the therapeutic effects of acupuncture and TCM in the treatment of primary dysmenorrhea through the included literature and found that acupuncture has more advantages than TCM in the treatment of primary dysmenorrhea.

The primary pathogenesis is "if obstructed, then painful," "if not vibrant, then painful." TCM has an excellent curative effect in treating primary dysmenorrhea with few adverse reactions. Chinese medicine pays attention to syndrome differentiation and treatment. According to the different manifestations, inducements, and physiques of different patients, the corresponding decoction, acupuncture, and other treatments are given. It can take care of qi and blood, viscera, and conform to nature, to achieve the purpose of dredging, replenishing the middle and replenishing qi.

This article only included seven clinical trials, with 492 primary dysmenorrhea patients. With this smaller sample size, the evidence power of this study was limited. This study still has some other limitations, mainly including: (I) the quality of the included literature was low, and the research methods including random allocation, blinding, description of loss to follow-up, and the withdrawal was not used or described. (II) Most studies have shortcomings, including small sample size, imperfect outcome indicators, and not paying attention to long-term efficacy. Therefore, the superiority of acupuncture versus TCM in the treatment of primary dysmenorrhea needs to be further verified by high-quality large-sample clinical studies. The design and implementation of future research should strictly follow the requirements of randomized controlled trials, with hidden grouping and perfect randomization to avoid selection bias; blinding methods should be applied as much as possible to avoid measurement bias and implementation bias.

\section{Acknowledgments}

Funding: None.

\section{Footnote}

Reporting Checklist: The authors have completed the
PRISMA reporting checklist. Available at http://dx.doi. org/10.21037/apm-20-1734

Conflicts of Interest: All authors have completed the ICMJE uniform disclosure form (available at http://dx.doi. org/10.21037/apm-20-1734). The authors have no conflicts of interest to declare.

Ethical Statement: The authors are accountable for all aspects of the work in ensuring that questions related to the accuracy or integrity of any part of the work are appropriately investigated and resolved.

Open Access Statement: This is an Open Access article distributed in accordance with the Creative Commons Attribution-NonCommercial-NoDerivs 4.0 International License (CC BY-NC-ND 4.0), which permits the noncommercial replication and distribution of the article with the strict proviso that no changes or edits are made and the original work is properly cited (including links to both the formal publication through the relevant DOI and the license). See: https://creativecommons.org/licenses/by-nc-nd/4.0/.

\section{References}

1. Ryan SA. The Treatment of Dysmenorrhea. Pediatr Clin North Am 2017;64:331-42.

2. De Sanctis V, Soliman AT, Elsedfy H, et al. Dysmenorrhea in adolescents and young adults: a review in different country. Acta Biomed 2016;87:233-46.

3. Ferrero S, Scala C, Vellone VG, et al. Transvaginal ultrasound-guided biopsy of adenomyosis. Ann Transl Med 2019;7:S341.

4. Kho KA, Shields JK. Diagnosis and Management of Primary Dysmenorrhea. JAMA 2020;323:268-9.

5. Ding M, Qiu YX, Yuan J. Comparative Observation on the Efficacy of Intradermal Auricular Acupuncture and Drugs on Dysmenorrhea. Chinese Acuponcture \& Moxibustion 1998;(12):25-6.

6. Fang F, Guo L, Liu YT, et al. Observation on the clinical effect of fire-acupuncture at Baliu points on primary dysmenorrhea. China Journal of Traditional Chinese Medicine and Pharmacy 2016;31:2406-8.

7. Zhang LK. Acupuncture Therapy Dysmenorrhea Randomized Controlled Observation. Journal of Practical Traditional Chinese Internal Medicine 2013;27:120-1.

8. Wang Y, Zeng XC. Observation on 35 Cases of Primary Dysmenorrhea Treated by Acupuncture. Journal of 
Practical Traditional Chinese Medicine 2008;24:302-3.

9. Liu MG, Zhang XN, Qu Y, et al. Clinical Observation on 60 Cases of Dysmenorrhea Treated by Acupuncture. Jilin Journal of Traditional Chinese Medicine 2013;33:514-5.

10. Wang HY. Treatment of 68 Cases of Primary Dysmenorrhea by Embedding Needle at Ear Points. Zhejiang Journal of Traditional Chinese Medicine

Cite this article as: Lin J, Liao W, Mo Q, Yang P, Chen X, Wang X, Huang X, Lu D, Ma M, He F, Wu P, Li K, Liang W, Tang H. A systematic review of the efficacy comparison of acupuncture and traditional Chinese medicine in the treatment of primary dysmenorrhea. Ann Palliat Med 2020;9(5):3288-3292. doi: 10.21037/apm-20-1734
2002;37:249.

11. Leng JQ, Lin Q, Hu YL. The effect of eye acupuncture for patients with $\mathrm{PD}$ on hemorheology. Clinical Journal of Chinese Medicine 2012;4:4-6.

(English Language Editor: J. Chapnick) 